\title{
Mouse colorectal cancer an early detection approach using nonlinear microscopy ${ }^{1}$
}

\author{
Mariana Bianchi $^{\text {a }}$, Javier Adur ${ }^{\mathrm{a}, \mathrm{b}}$, Silvana Y. Ruff ${ }^{\mathrm{a}}$, María F. Izaguirre ${ }^{\mathrm{a}}$, Hernandes F. \\ Carvalho ${ }^{\mathrm{c}}$, Carlos L. Cesar ${ }^{\mathrm{c}}$ and Víctor H. Casco ${ }^{\mathrm{a},{ }^{*}}$ \\ ${ }^{a}$ Microscopy Laboratory Applied to Molecular and Cellular Studies, Engineering School, National \\ University of Entre Rios, (3101) Oro Verde, Entre Rios, Argentina. \\ ${ }^{b}$ Research and Transfer Center of Entre Rios (CITER) CONICET-UNER (3101) Oro Verde. Entre \\ Ríos. Argentina. \\ ${ }^{c}$ INFABiC - National Institute of Science and Technology on Photonics Applied to Cell Biology, \\ Campinas, São Paulo, Brazil.
}

\begin{abstract}
Murine induced colon cancer has been used to demonstrate that Second Harmonic Generation (SHG) microscopy images, combined with Two-Photon Excitation Fluorescence (TPEF) and specific quantization scoring methods allow distinguishing early alterations in colon mucosa. TPEF was used only to identified crypts and submucosa regions, whereas the image analysis was used to get quantitative data (Integrated Intensity and Aspect Ratio scoring) of different cancer stages. The submucosa amount of collagen fibers was significant and their orientation suffering proportional changes with the development of the pathological processes. Both after the fourth and eighth weeks after colon cancer induction, integrated intensity and aspect ratio values have shown significant statistical differences compared with control samples. Thus, SHG microscopy has proved to be a useful quantitative tool to highlight early changes of submucosa and the progression of these through the cancer development.
\end{abstract}

Keywords: Colon cancer, early detection, second harmonic generation, two-photon excitation fluorescence

\section{Introduction}

Colon cancer is one of the leading causes of death in humans [1]. During the last decade, the scientific community has undertaken this challenging job of discovering the novel and efficient method of using of dyes, magnification endoscopy, low bandwidth imaging as well as endogen fluorescence imaging for colon cancer diagnosis [2-4]. Even with the high cost and risk associated with the endoscopic biopsy hematoxylin and eosin stained (H\&E), it is still the golden standard for local screening. Due to the various drawbacks of this technique such as high cost, cumbersome and excisional process, there was a need for detecting precursor lesions based on the new imaging technology.

\footnotetext{
${ }^{1}$ These authors contributed equally to this work.

${ }^{*}$ Corresponding author: Víctor H. Casco, Microscopy Laboratory Applied to Molecular and Cellular Studies, Engineering School, National University of Entre Rios, (3101) Oro Verde, Entre Rios, Argentina. E-mail: vcasco@bioingenieria.edu.ar
}

0959-2989/14/\$27.50 @ 2014 - IOS Press and the authors. 
Recognition of premature cancer injury is an open problem, which has not yet been solved. In this regard, non-invasive approach is helpful for colon cancer detection. Examples of these techniques are fluorescence spectroscopy, Raman spectroscopy, and non-linear optical (NLO) microscopies, such as Two-photon imaging and Second Harmonic Generation (SHG) microscopy [5-7]. The last approach is a predominant imaging technology with advantages over confocal and other current imaging technologies $[8,9]$. Interestingly, SHG can directly visualize the collagen assembly in the extracellular matrix (ECM) without the requirement of any exogenous staining [10]. Subsequently, it has been observed that changes in the ECM can be used as substitute markers for differentiating malignant from nonmalignant tissue $[11,12]$.

This work was used an murine model of induced colon cancer, in which the intestinal adenocarcinomas grow rapidly and their histological transformations remember those encountered in human body, to demonstrate that SHG microscopy images in combination with specific quantification scoring methods allow to discriminate early alterations in colonic mucosa. Finally, we can say that, this methodology could be used as a non-invasive alternative for early colon cancer detection.

\section{Methods}

\subsection{Murine model of chemical-induced colon cancer (AOM/DSS)}

Adult male BALB/c mice $(\mathrm{n}=12) 20$ - $30 \mathrm{~g}$ body weight, were purchased from Center of Biological Experimentations and Biotery of National University of Litoral, Santa Fe. Argentina. Mice were accommodated in cages (4/cage) with clean water and food, under controlled conditions of temperature, photoperiod, and wetness. They were isolated during the first 7 days, arranged in random order by weight into experimental and control groups. Experimental animals were intraperitoneally injected with Azoxymethane (AOM) $10 \mathrm{mg} / \mathrm{Kg}$ body weight and a week later, they were given Dextran Sodium Sulphate (DSS) in the beverage water for seven days, following the procedure reviewed by Tanaka and coworkers [13]. Control animal were injected with saline and were not given DSS. Samples were taken at day zero, fourth and eighth weeks after injection. Colon segments were destined to histological processing (for control proposes) and nonlinear optical microscopy observation (for quantification).

\subsection{Nonlinear microscopy setup and image acquisition}

Figure 1A depicts a diagram of the instrument used. The system was built around an inverted microscope which was recently described in detail [14]. The polarization at the sample was not linear because the optical elements of the setup introduce some degree of ellipticity on the incident beam. Furthermore, in this type of sample (high scattering) may help to visualize fibrils in all directions. TPEF and SHG signals were detected in transmitted mode. The transmitted SHG $(470 \mathrm{~nm})$ and TPF (>490 $\mathrm{nm}$ ) signals were condensed by the lens $0.55 \mathrm{NA}-\mathrm{WD} 26 \mathrm{~mm}$ (Carl Zeiss) and acquired at the same time by non-descanned detector (NDD) using a filter SP720 (Omega Filters) to block back-reflected laser light. To avoid useless fluorescence light from the SHG channel a filter cube (dichroic LP490 nm and filter SP485 nm) was positioned in front of the SHG detector. To collect TPEF signal, a filter cube (mirror and filter BP540 nm) was used. The figure 1B exemplifies two representative images (1024 x 1024 pixel spatial resolution) of crypts and submucosa regions, acquired with this setup, displaying TPEF (green) + SHG (red) images. 


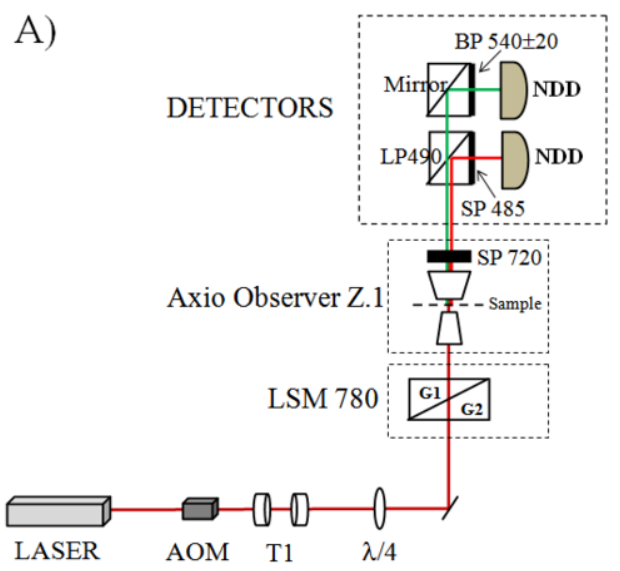

B)

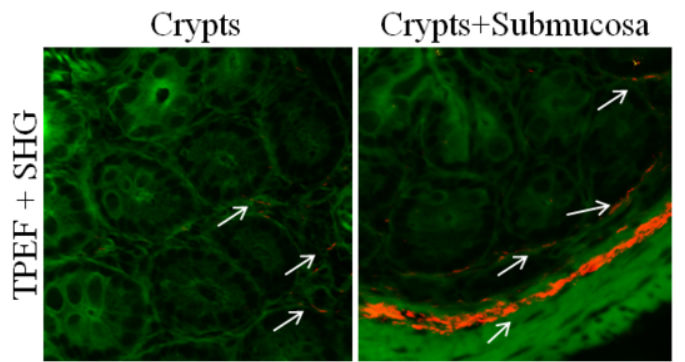

Figure 1. A) Experimental setup to TPEF and SHG microscopy based on an inverted microscope Axio Observer Z.1 and LSM 780-NLO confocal scan head (Carl Zeiss AG, Germany), AOM: acoust-optic modulator, T1: telescope, $\lambda / 4$ : quarter waveplate, G1/G2: galvanometer mirrors, SP: short-pass filter, BP: bandpass filter, LP: long-pass filter, NDD: Non Descanned Detector. B) Combination of TPEF and SHG images of colon crypts and crypts + submucosa. The arrows point the collagen fibers (red).

\subsection{Scoring methods}

All methods were carried out with ImageJ (v1.45) software (NIH) and calculations were made for 21 regions of interest (ROI) (300 x 300 pixels) chosen over selected images. For SHG intensity analysis, signal was separated from the background with a threshold at level 30 from the 0 to 255 gray levels. From each ROI the integrated density (ID) was quantified. To achieve the aspect ratio (AR), Fast Fourier Transform (FFT) was used, which was proved to be efficient to consign the degree of image organization. If the fibers have a parallel arrangement, the intensity plot of the FFT image look as an ellipse and consequently will have a higher AR. In contrast, fiber with aleatory arrangement, exhibit an intensity plot of the FFT image, closer to the shape of a circle. Anisotropy calculations were performed using an ellipse fitting on the threshold FFT images and the aspect ratio was obtained based on the calculation of the ratio between short and long axes of the ellipse [15]. Anisotropic sample gives an AR close to 0, whereas more isotropic sample gives a value of AR close to 1 . These scoring were applied in crypts and submucosa images. To obtain, a qualitative observation of the presence and angle of collagen fibers, a three-dimensional representation was performed using the maximum projection of intensity (MPI) from a stack of $46 \mu \mathrm{m}$ thick.

\subsection{Statistical analysis}

One-way analysis of variance (ANOVA) with a post-hoc Tukey-Kramer test was used. The $\mathrm{p}<0.05$ were considered significant $(*)$ whereas $p<0.01$ were considered very significant $(* *)$. Data were analyzed with SPSS 10.0 software. 


\section{Results}

\subsection{Cancer colon development}

In the animal model, the control mouse colon exhibits a crypt-architecture and an ordered cell polarity pattern. The epithelium contains little, orbed, pale basal nuclei and distinct goblet and absorptive cell phenotypes (Fig. 2A). During week 4 of AOM/DSS treatment, colon lesions are appeared, which characterize the first step of the pathology, in the form of the aberrant crypt foci (ACF). On the right of the image (Fig. 2B), an ACF whit fused crypts and low grade of dysplasia can be observed, where most of the nuclei retain their cell polarity. On the left of the image (Fig. 2B), abundant lymphocyte infiltration can be visualized, possibly, due to the inflammation process. During week 8 after the injection, the lesions transform into a high grade of dysplasia. Dysplastic epithelium contains longer, packed and dark-staining nuclei. Secondly, it is observed that the crypts architecture have been lost, and depicted on the right of the image which is fused and round in shape and also on the left, long and slender villi, with tapered tips (Fig. 2C).

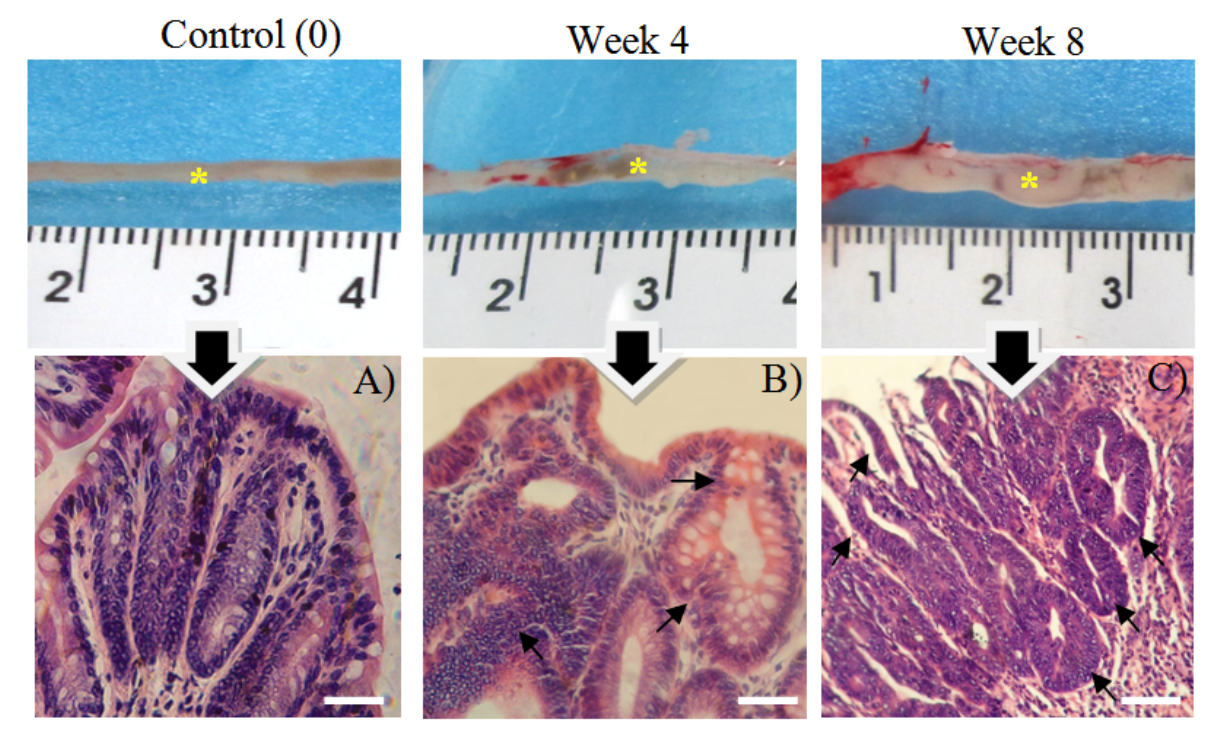

Figure 2. Colon cancer development after AOM injection. Macroscopic view of murine colon segments and H\&E stained sections taken from the regions labeled by yellow asterisk. Scale bars: $50 \mu \mathrm{m}$.

\subsection{Early cancer discrimination by $S H G$ microscopy analysis}

Crypt-cross sections view allow perceiving the circular arrangement configuration, typical of control colonic crypts, identified by columnar cells and intercalated goblet cells. TPEF images (green) shows crypts with rounded-luminal openings, characteristic of normal pattern of colon mucosa (Fig. 3). SHG images (red) exclusively delineate the collagen scaffold within lamina propria. The combination of TPEF and SHG images enables a superior evaluation of the spatial organization of the tissues. 


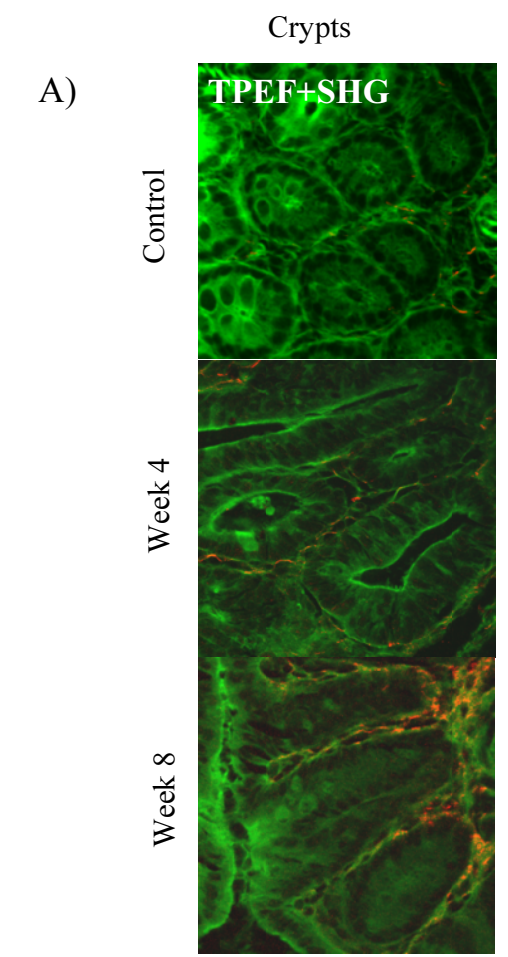

B)

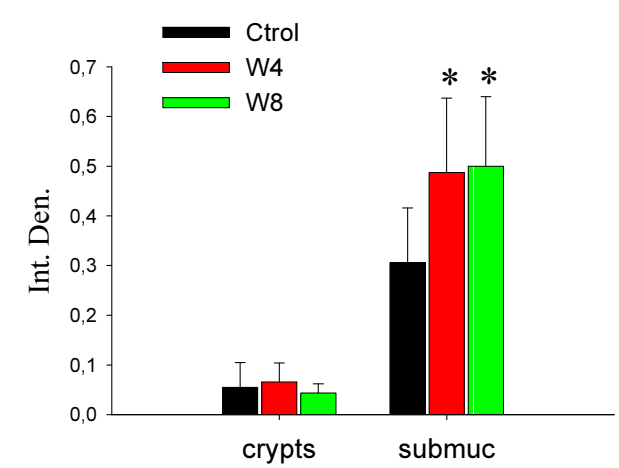

Submucosa

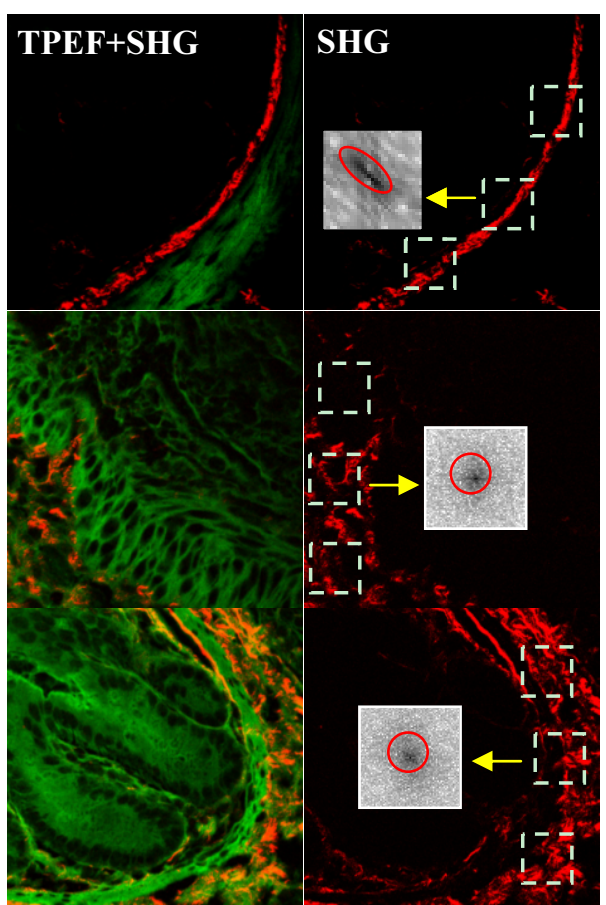

C)

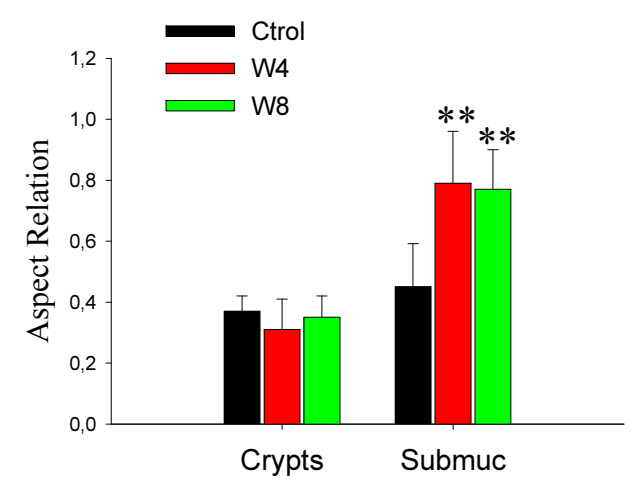

Figure 3. A) TPEF (green) and SHG (red) images of mouse colon. In SHG images to simplify one ROI (white squared) shows (in the inset) the corresponding FFT image. Scale bars $=50 \mu \mathrm{m}$. B) The integrated density of colon samples averaged on all the examined ROI. In total, twenty one ROI (300 x 300 pixel side squared) were selected for control, week 4, and week 8 samples). C) Histograms of the AR averaged on all the examined ROI. In total, twenty one ROI ( $300 \times 300$ pixel side squared) were selected for control, week 4 , and week 8 samples. ${ }^{*}(p<0.05) ; * *(p<0.01)$.

Using SHG signal, the amount and distribution of the collagen fibers were quantified at subsequent weeks, after the post AOM injection. In crypts no differences in SHG signal were detected (left column of Fig 3A) but in submucosa, the amount of collagen fibers was augmented in proportion with the development of the pathology (middle and right column of Fig. 3A). Figure 3B indicates the average of ID from all examined samples. Whereas week 4 and 8 post AOM injection ID were significantly 

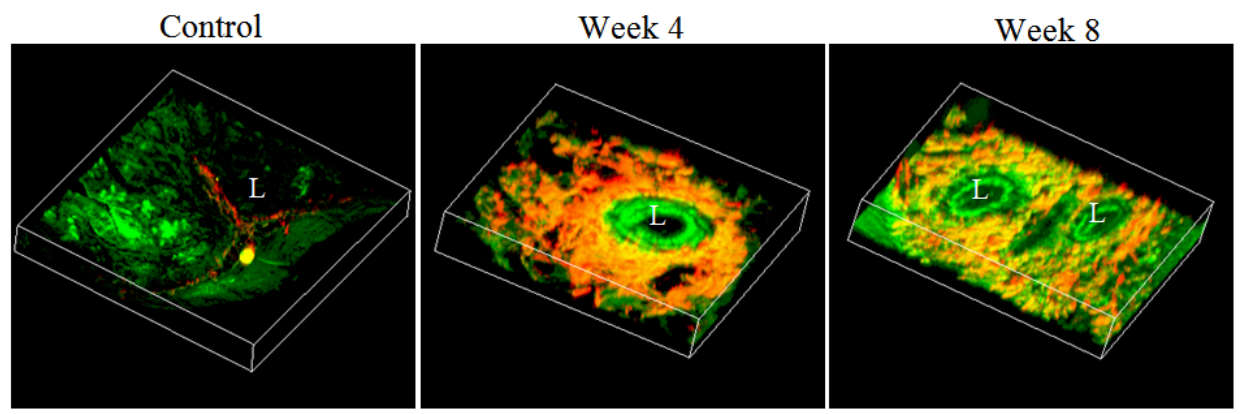

Figure 4. Maximum projection (TPEF+SHG) of individual crypts. 46 images separated $1 \mu \mathrm{m}$ each $(512 \times 512 \times 46)$. L: luminal crypt orifice.

higher $(\mathrm{p}<0.05)$ compared with control samples. Regarding the arrangement of the collagen fibers, anisotropy calculations were estimated (Fig. 3C). FFT images of control sample exhibit a more elliptical profile (fibers are arranged in parallel form) when compared to the FFT images of samples process with AOM, which presents a more circular configuration (fiber without any specific arrangement). The figure $3 \mathrm{C}$ shows the averaged AR of the examined stages. Again, at week 4 and 8 , AR values were increased and which was extremely significant $(\mathrm{p}<0.01)$, compared to normal colon. These data helps to verify the fact that, normal submucosa is a better ordered tissue than the transformed one. The three-dimensional representations (Fig. 4) show previous observation that collagen fibrils adopt a distinctive behavior relative to epithelium, during the pathological process.

\section{Discussion and Conclusions}

In a previously reported work of our group, the mucosa and submucosa layers in normal human rectum were imaged by SHG and TPEF [16]. Along with other components and structures, the content and distribution of collagen fibers was reported in [16], also highlighted the benefits of this technique as a new promising method of early rectal cancer detection. In another study we have proposed how to distinguish between normal and high grade of dysplasia and cancer, using SHG images of human colon [17], but the early stages of the pathology were not evaluated.

The present study demonstrates that SHG microscopy allows detecting early changes of colonic submucosa in an animal model of colorectal cancer. The chemical carcinogen AOM effectively reproduced the alterations found in human colon cancer. While TPEF images contributed to the understanding of the tissue microstructures, SHG microscopy enabled the identification of two collagen features (amount variation and fibers orientation), which exhibited significant differences at different colonic cancer stages. With the help of these data, it was possible to gain quantitative information (ID and AR scoring) in different cancer stages. In agreement with our former results, previous reports in human colon and other tissues suggest that the epithelial cells preferentially invade tissues where the collagen fibers became perpendicularly aligned, instead of randomly arranged ones $[5,17,19]$. Thus, SHG microscopy proves to be a powerful quantitative tool which is combined with specifics gene tumor classification algorithms $[20,21]$, could allow detecting early changes of submucosa and their progression through the cancer expansion.

SHG has the ability to image tissues in situ, without any exogenous contrast label and avoiding the bleaching effects of the fluorescence methods. The fact that SHG could reach high penetration, main- 
taining appropriate resolution levels allowing 3D sectioning of the sample, make possible to postulate it, as a future endoscopic tool for 3D in vivo non-invasive optical biopsy tool.

Finally, we can say that these results addresses the promising benefits of SHG imaging for clinical purposes, and as a tool for biological and medical research to study the dynamics of the extracellular matrix modifications. With the ability of quantifying the submucosa alterations, as shown in this work, it is expected that a SHG-based endoscopic tool could assist in vivo studies and help us to reach an early objective cancer diagnosis method in the near future.

\section{References}

[1] A. Jemal, R. Siegel, J. Xu, and E. Ward, Cancer statistics 2010. CA Cancer J Clin 60 (2010), 277-300.

[2] K. Imaizumi, Y. Harada, N. Wakabayashi, Y. Yamaoka, H. Konishi, P. Dai, H. Tanaka, and T. Takamatsu, Dualwavelength excitation of mucosal autofluorescence for precise detection of diminutive colonic adenomas. Gastrointest. Endosc. 75 (2012), 110-117.

[3] P.C. Lopes, J.A. Moreira, A. Almeida, A. Esteves, I. Gregora, M. Ledinsky, J.M. Lopes, R. Henrique, and A. Oliveira, Discriminating adenocarcinoma from normal colonic mucosa through deconvolution of Raman spectra. J. Biomed. Opt. 16 (2011), 127001.

[4] B. Banerjee, T. Renkoski, L.R. Graves, N.S. Rial, V.L. Tsikitis, V. Nfonsam, J. Pugh, P. Tiwari, H. Gavini, and U. Utzinger, Tryptophan autofluorescence imaging of neoplasms of the human colon. J. Biomed. Opt. 17 (2012), 016003.

[5] S. Zhuo, X. Zhu, G. Wu, J. Chen, and S. Xie, Quantitative biomarkers of colonic dysplasia based on intrinsic second harmonic generation signal, J. Biomed. Opt. 16(2011), 120501.

[6] R. Carriles, D.N. Schafer, K.E. Sheetz, J.J. Field, R. Cisek, V. Barzda, A.W. Sylvester, and J.A. Squier, Imaging techniques for harmonic and multiphoton absorption fluorescence microscopy. Rev. Sci. Instrum. 80(2009), 081101.

[7] R. Cicchi, A. Sturiale, G. Nesi, F. Tonelli, and F.S. Pavone, Two-photon imaging and spectroscopy of fresh human colon biopsies. Proc. SPIE Vol. 8226 (2012), 82263S, 10.1117/12.907822.

[8] M. Wang, K.M. Reiser, and A. Knoesen, Spectral moment invariant analysis of disorder in polarization-modulated second-harmonicgeneration images obtained from collagen assemblies. J Opt Soc Am A Opt Image Sci Vis. 24 (2007), 3573-3586.

[9] G. Cox, E. Kable, A. Jones, I. Fraser, F. Manconi, and M.D. Gorrell, 3-dimensional imaging of collagen using second harmonic generation. J Struct Biol. 14 (2003), 53-62.

[10] P. Campagnola, Second harmonic generation imaging microscopy: applications to diseases diagnostics. Anal Chem. 83 (2011), 3224-3231.

[11] O. Nadiarnykh, R.B. LaComb, M.A. Brewer, and P.J. Campagnola, Alterations of the extracellular matrix in ovarian cancer studied by second harmonic generation imaging microscopy. BMC Cancer. 10 (2010), 94.

[12] V. Ajeti, O. Nadiarnykh, S.M. Ponik, P.J. Keely, K.W. Eliceiri, and P.J. Campagnola, Structural changes in mixed col I/Col V collagen gels probed by SHG microscopy: implications for probing stromal alterations in human breast cancer. Biomed Opt Express. 2 (2011), 2307-2316.

[13] T. Tanaka, Colorectal carcinogenesis: Review of human and experimental animal studies. J. Carcinog. 8(2009), 1-19.

[14] J. Adur, V.B. Pelegati, A.A. de Thomaz, M.O Baratti, L.A. Andrade, H.F. Carvalho, F. Bottcher-Luiz, C.L. Cesar, Second harmonic generation microscopy as a powerful diagnostic imaging modality for human ovarian cancer. J Biophotonics. 7 (2014), 37- 48.

[15] P. Matteini, F. Ratto, F. Rossi, R. Cicchi, C. Stringari, D. Kapsokalyvas, F. S. Pavone, and R. Pini, Photothermallyinduced disordered patterns of corneal collagen revealed by SHG imaging. Opt. Express 17 (2009), 4868-4878.

[16] N. Liu, G. Chen, J. Chen, J. Yan, S. Zhuo, L. Zheng, and X. Jiang, Multiphoton microscopic imaging of normal human rectum tissue. Scanning $\mathbf{6}$ (2010), 347-350.

[17] J. W. Birk, M Tadros, K. Moezardalan, O. Nadyarnykh, F. Forouhar, J. Anderson and P. Campagnola, Second Harmonic Generation Imaging Distinguishes Both High Grade Dysplasia and Cancer from Normal Colonic Mucosa. Dig Dis Sci (2014), 1-6.

[18] J. Adur, V. B. Pelegati, A. A. de Thomaz, M. O. Baratti, D. B. Almeida, L. A. L. A. Andrade, F. Bottcher-Luiz, H. F. Carvalho, C. L. Cesar, Optical Biomarkers of Serous and Mucinous Human Ovarian Tumor Assessed with Nonlinear Optics Microscopies. PLoS ONE 7 (2012), e47007.

[19] M. W. Conklin, J. C. Eickhoff, K. M. Riching, C. A. Pehlke, K. W. Eliceiri, P. P. Provenzano, A. Friedl, P. J. Keely, Aligned collagen is a prognostic signature for survival in human breast carcinoma. Am J Pathol. 178 (2011), 1221-32. 
[20] H. Wang, S. Zhang, Tumor classification based on orthogonal linear discriminant analysis. Bio-Medical Materials and Engineering 24 (2014) 1399-1405.

[21] L. Sun, J. Xu, Feature selection using mutual information based uncertainty measures for tumor classification. BioMedical Materials and Engineering 24 (2014) 763-770.s 